\title{
CORRESPONDENCE
}

Correspondents are asked to be brief

Streptokinase and Myocardial Infarction M. Verstraete, M.D..............679

Treatment of Bell's Palsy

E. C. O. Jewesbury, F.R.C.P.; D. Taverner,

F.R.C.P. $\quad \ldots \ldots \ldots \ldots \ldots \ldots \ldots \ldots \ldots 696 \ldots \ldots$

Lead in the Community

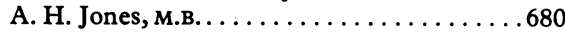

Health Care in the U.S.A.

Max H. Parrott, M.D. . . . . . . . . . . . . 6680

Psychosis and Ketamine

J. W. Dundee, M.D., F.F.A. R.C.S., and D. L.

Coppel, F.F.A. R.C.s.I. ..........681

Age and Carcinoma of the Cervix

A. F. Anderson, F.R.C.o.G..........681

Herpetic Whitlows: A Medical Risk

B. E. Juel-Jensen, B.M. . . . . . . . . . .681

Compression of Coeliac Axis

J. H. Swallow, M.R.C.P.... . . . . . 682
Screening for Spastics

Taste and Smell

J. Shafar, F.R.C.P................688

Myxoedema during Cyclophosphamide Therapy

V. J. Coffey, M.B., D.M.R...........6682

Coronary Deaths-How Unexpected?

J. H. Lacey, M.B. . . . . . . . . . . . . . . . . . 684

Incidence of Gonorrhoea

A. Richens, PH.D., M.R.C.P., and D. J. F.

Rowe, B.Sc....................684

Responsibility of the Scientist

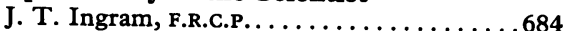

Dichloralphenazone and Breast Milk

J. O'Grady, м.в..............

Need for Asylums

Freda S. Reed, D.P.M.

Unfair Pressure for Abortion

H. C. McLaren, F.R.C.o.G.

A. W. Clare, M.B., and J. Hamilton, M.B. . . .685

Trends in Specialization

E. N. Wardle, M.R.C.P............685

Antigen in Nigerian Blood

Donors and School Children

T. I. Francis, F.R.C.P.ED., and J. A. Smith,

M.B. ......................683

Scientific Basis of Clinical Practice

R. R. Macdonald, F.R.C.o.G... . . . . . . . 685

Single-handed Rural

G. Scott, M.D.................6685

\section{Streptokinase and Myocardial Infarction}

SIR,-Dr. R. Heikinheimo (6 November, p. 361 ), in commenting on the report of the European Working Party on thrombolytic treatment in recent myocardial infarction (7 August, p. 325), remarks that more patients with recurrent infarction (44 of 357) belonged to the heparin treated group than the streptokinase treated group (35 of 373). This difference is, however, not significant $(P=0.066)$, in contrast to the prevalence of arrhythmia on admission (10 of 373 streptokinase treated patients compared to 77 of 357 heparin treated patients).

It is indeed hard to resist the suspicion that there was some systematic reason why the eight deaths which occurred before the start of the infusion were all in the heparin group. An obvious explanation would be that there was, on average, more delay in starting heparin than streptokinase. This point was carefully checked; the time interval between admission to hospital and the start of the infusion was found to be similar in the two treatment groups. Moreover, the eight patients who died before the start of treatment were admitted to four different co-operating medical centres and were spread over the total duration of the trial. Six of them were admitted in shock. This trial is certainly a saga of great misfortune in that all preinfusion deaths were in the heparin group.

However, considering hospital mortality after the 24-hour infusion, there were 36 deaths in 340 streptokinase-treated patients $(10.6 \%)$ versus 57 deaths in 320 heparintreated patients $(17.8 \%)$; this difference is significant, $P$ two-tailed being 0.011 (and not 0.046 as stated in Dr. Heikinheimo's letter, who confused the overall death rate with an analysis of subgroups).

It is logical to expect some relationship between a favourable response to thrombolytic treatment and the time interval be- tween the onset of symptoms of myocardial infarction. In the report of the European Working Party no significant difference between the two treatment groups was obtained when the patients were retrospectively grouped according to the time-interval between the onset of acute symptoms and the start of the infusion. In the first 12 hours subgroup the hospital mortality (21 days) was $17 \cdot 1 \%$ in the streptokinase group versus $20.2 \%$ in the heparin group; in the 12 to 24 hours subgroup the corresponding figures were $22.7 \%$ versus $29 \cdot 1 \%$. These results are at variance with other trials conducted in patients with myocardial infarction admitted to general wards. ${ }^{12}$

The weight of Dr. Heikinheimo's criticism is that though all patients received oral anticoagulants at the start of treatment the control group had a heparin infusion lasting as long (namely 24 hours) as the streptokinase infusion in the experimental group. The report clearly states that, at the time the European trial was planned, it was still the prevailing view that oral anticoagulants supplemented with heparin for the first few days was part of the conventional treatment in acute myocardial infarction. This opinion apparently was shared also by Dr. Heikinheimo, since one of the four treatment centres of the joint trial on thrombolytic treatment in myocardial infarction was coordinated in Finland by him in which oral anticoagulants and heparin were administered simultaneously to the experimental and control groups. ${ }^{3}$ More recently serious doubts have been raised as to the validity of using combined oral and intravenous (heparin) anticoagulants in these patients; this problem is being discussed elsewhere."

Dr. Heikinheimo's final remark, which alleges that the rules of common sense were broken and that streptokinase is now recommended for recent myocardial infarction, seems unfair. The discussion of the report indeed clearly indicates that the present evidence of the benefit of streptokinase in patients with acute myocardial infarction is too frail to permit a broad recommendation. Further stringent experimentation in patients with recent myocardial infarction admitted to general wards and to coronary care units should therefore be conducted by noncommitted groups. There is also an ethical motivation for clinical investigators who are engaged in this field not to leave an issue of this importance unsettled in order to avoid imprecise usage of thrombolysis based on incomplete evidence.-I am, etc.,

\section{Verstraete}

European Working Party Co-ordinating Centre, Academisch Ziekenhuis,

Leuven,
Belgium

Schmutzler, R., et al., Deutsche Medizinischo Wochenschrift, 1966, al., Deutsche Wochenschrift, 1966, 91,581 . Haematology, Abstracts Mternational Congress of Heikinheimo, R., et al., Acta Medica Scan dinavica, 1971, 189, 7 .

4 Verstraete, M., Angiologica, 1971, 8, 43.

\section{Treatment of Bell's Palsy}

SIR,-Dr. D. Taverner with various colleagues has contributed much to our understanding of the factors governing the prognosis and treatment of Bell's palsy. In the latest paper (2 October, p. 20) Dr Taverner and his colleagues conclude, "At present we believe that oral prednisolone is the treatment of choice in Bell's palsy and should be given in full doses from the day of onset." A nine-day course, starting with $80 \mathrm{mg}$ daily for the first five days, is advised.

The practical problem arises, "Which patients with Bell's palsy should be so treated?" The possible side effects of prednisolone in certain patients have already 
been mentioned by Dr. W. T. Berrill (13 November, p. 425) and it is well known that the prognosis for spontaneous recovery from Bell's palsy is reasonably good. Taverner and his colleagues ${ }^{1}$ in describing the natural history of Bell's palsy comment that about three-fifths of patients recover completely. They state that of the remainder, who develop degeneration of the facial nerve, about one-quarter remain permanently dissatisfied with their eventual state of recovery. Thus some $10 \%$ of patients who develop Bell's palsy may be expected to have persisting paralysis and disfigurement, often with troublesome permanent sequelae.

In an earlier paper Langworth and Taverner $^{2}$ referred to a group of 39 patients with Bell's palsy in whom partial denervation had occurred. No treatment was given, and all patients in this group, who were followed up for nine months, recovered at least $80 \%$ of normal function. Twenty-nine patients in whom complete denervation occurred were also described, and clinical recovery of the circumoral muscles varied from almost nil to $40 \%$ even in this group.

No treatment is necessary if no denervation occurs, but it is notoriously difficult or impossible by electrical tests to identify within the first few days of the palsy those cases in which nerve degeneration is developing and who might be helped by steroid therapy. From the clinical aspect, if the facial palsy is complete at the onset or within the first few days full recovery cannot be expected. Unilateral loss of taste sensibility or marked impairment of the anodal galvanic stimulation response by the anterior two-thirds of the tongue during the first 14 days of the palsy is also held to imply a bad prognosis. According to Matthews ${ }^{3}$ an absolutely good prognosis, with complete recovery, is indicated if the facial palsy remains partial within six days of the onset. As Miller has also observed: "If any voluntary movement whatever is present five days after the onset, functional recovery will be nearly perfect, even where some evidence of denervation and reinnervation remains."

Taverner and his colleagues 156 believe that in very severe attacks of Bell's palsy the major damage may be done within the first few hours and that treatment should therefore be started at once. If steroids prevent or reduce nerve degeneration, and if they must be given before such degeneration can be demonstrated, then treatment must begin when the palsy first appears. Indeed, Briggs and Potter $^{7}$ have used steroid prophylactically in injured patients who had radiological evidence of a petrous temporal fracture and a bleeding ear on the same side. An eight-day course of ACTH gel was given, the first dose being administered on the day of the injury. Without such treatment $38 \%$ of patients develop some degree of facial weakness but with steroid prophylactic therapy only $8 \%$ did so. Nevertheless, they are not convinced that such prophylactic treatment need always be adopted, since most patients who develop this complication of head injury will recover completely or to an acceptable degree from their facial weakness without treatment.

Whether the increased recovery rate which is claimed in Bell's palsy is sufficient to justify the use of full doses of prednisolone in every case may still be regarded as a controversial question. In about $80 \%$ of the patients it is unnecessary and steroid therapy still provides no guarantee of full recovery. Treatment must in any event be given at the earliest possible moment after the onset of the palsy. If persistent palsy is preventable it must be prevented, but are we to regard the immediate use of predinsolone as obligatory in all cases of Bell's palsy? I am, etc.,

ERIC C. O. JeWESBURY

Royal Northern Hospital, 1 raverner, D., Fearnley, M. E., Kemble, F., Medical Fourmal, 1966, 1, 391.
Langworth, E. P., and Taverner, D., Brain, 1963, 86, 465. P., and Taverner, D., Brain,
Matthews, W. B., British Medical foumal, 1961, 2.,215. 3, 815

5 Taverner, D., Kemble, F., and Cohen, S. B. British Medical Fournal, 1967, 4. 581 . 6 Taverner, D., Cohen, S. B., and Hutchinson, B. C., British Medical fournal, $1971,4,20$.
Briggs, M., and Potter, J. M., British Medical fournal, 1971, 3, 458 .

SIR,-Dr. W. T. Berrill in his letter (13 November, p. 425) refers to a recent pape by my colleagues and me ( 2 October, $p$. 20).

We explained that the trial we reported was designed in the expectation of showing that corticotrophin is superior to prednisolone in the management of Bell's palsy. Anticipating possible objections, we deliberately chose a dose of corticotrophin already known to be effective in this situation ${ }^{2}$ and as large a dose of prednisolone as seemed prudent yet likely to be effective. The incidence of side effects was low and is fully reported. Since completing the trial we have reduced the initial dose of prednisolone to $60 \mathrm{mg}$ daily without any apparent deterioration in our results.

We showed that prednisolone achieved better results than corticotrophin when treatment was started during the first two days after onset $(0.025>P>0.01)$. The difference in the proportions quoted by Dr. Berrill is not significant $(P>0 \cdot 10)$.

Sex has no bearing on the outcome in Bell's palsy whether treated or not. I have today examined a previous analysis of 340 patients similar to our present series and fully confirmed this statement. The details of gender were not included in our report because of their irrelevance. They are shown in the accompanying Table. None of the differences are significant $(P>0 \cdot 10)$

Sex Distribution of Patients with Bell's Palsy

\begin{tabular}{l|c|c|c|c|c}
\hline Sex & Total & $\begin{array}{c}\text { Re- } \\
\text { covered }\end{array}$ & $\begin{array}{c}\text { Dener- } \\
\text { vated }\end{array}$ & $\begin{array}{c}\text { Pred- } \\
\text { nisolone }\end{array}$ & $\begin{array}{c}\text { Cortico- } \\
\text { trophin }\end{array}$ \\
\hline Male & 97 & 73 & 24 & 42 & 55 \\
Female & 89 & 69 & 20 & 50 & 39
\end{tabular}

The reasons for exclusion of patients from the trial are fully stated. It would be an absurdity to use the results from such a heterogeneous group as a check on the validity of a fully randomized prospective controlled trial.-I am, etc.,

The General Infirmary,

D. TAVERNER

Leeds

1 Taverner, D., Cohen, S. B., Hutchinson, B. C., British Medical fournal, 1971, 4, 20. Taverner, D., Fearnley, M. E.' Kemble, F., Miles, D. W., and Peiris, Ó. A., British
Medical foumal, 1966, 1, 391.
Lead in the Community

SIR,-Recent discussions in the press and elsewhere of environmental lead problems encouraged me to read again your leading article on lead in air (18 September, p. 653). British Rail has an unfortunate inheritance of many thousands of iron and steel structures coated with lead paints for more than a century. The surface preparation of these structures for maintenance painting is costly, and our civil engineers have of necessity adopted techniques for the rapid removal of old, loose paint and corrosion residues. These methods involve burning off deposits with oxy-gas flames or removing them by mechanical abrasion. The lead hazard is obvious, and considerable effort and expense is devoted to its control.

Our search for alternatives to lead primers and surface coatings has proved remarkably successful. Non-lead paint systems totally replaced lead for the painting of rolling stock many years ago. No lead finishing coats are now used on wooden or iron and steel structures. The most intransigent problem is finding an alternative to the traditional red lead primer for new steelwork. Cautious engineers are reluctant to abandon well known methods of corrosion prevention on expensive iron and steel structures which have to last for decades. The problem has assumed added urgency because of the increasing application of the box girder, more particularly in other than railway structures.

There is an urgent need to adopt safe alternatives such as primers based on zinc phosphate. Zinc phosphate primers can be sprayed at high speed, and I am assured that subsequent coats of paint can be applied at a shorter interval after initial priming. The technical advantages are self-evident. British Rail have now decided that zinc phosphate primers will replace red lead for the treatment of all new steelwork and maintenance painting.-I am, etc.,

A. H. JONES

British Railways Board,

Marylebone Station

London N.W.1

\section{Health Care in the U.S.A.}

SIR,-I must take issue with a statement made in your leading article on "Health Care in the U.S.A." (18 September, p. 658). You stated that "The American Medical Association's Medicredit plan would provide only basic hospital and doctor care." Allow me to correct that assertion with a description of what Medicredit really does provide.

Medicredit provides comprehensive health care for all persons under age 65 through a voluntary national health insurance plan. It guards against both the ordinary and catastrophic expenses of illness. It would assure the poor of access to quality health care and help others purchase private health insurance through a sliding scale of tax credits.

Medicredit provides payment of expenses for inpatient care in a hospital or extended care facility; outpatient and emergency care; and physician's services wherever they are provided. Basic benefits in a 12-month policy period would include 60 days of inpatient hospital care, including all of the customary inpatient services such as semiprivate room staff nursing; drugs and oxygen; blood and 